Website: http://revistas.lamolina.edu.pe/index.php/acu/index

(C) Universidad Nacional Agraria La Molina, Lima - Perú

\title{
Génesis, morfología, clasificación y susceptibilidad de suelos de la parte media de la cuenca del río Abujao Región Ucayali
}

\author{
Genesis, morphology, classification and susceptibility soil part middle river \\ Abujao Region Ucayali \\ Euclides R. Ramírez Zumaeta ${ }^{1}$; Manuel F. Valencia Ramos ${ }^{2 *}$; Jorge W. Vela Alvarado ${ }^{3}$ \\ *Autor de correspondencia
}

\section{Resumen}

El objetivo de presente estudio fue obtener una fuente fiable de información para un manejo eficiente de los recursos naturales existente. Para ello, se caracterizó física, morfológicamente y taxonómicamente los suelos de la zona. La metodología empleada se basó en la Soil Survey Manual; y la clasificación taxonómica según Soil Taxonomy. También se utilizó el método multivariable para determinar la susceptibilidad de los suelos. Se identificaron 17 series de suelos, uno del Orden Entisols, trece Inceptisols y tres Ultisols; asimismo, mediante el análisis mineralógico se observó que la presencia del mineral albita (feldespato) predispone a que esos suelos sean los menos desarrollados dentro de su orden, y que los suelos en que se observa la presencia de rutilo (oxido de titanio) suelos muy evolucionados. Aquellos suelos que presentan arcillas como la montmorillonita y la illíta son suelos con un desarrollo moderado. Con respecto a la susceptibilidad de la tierra, la zona en estudio presenta tres niveles: Ligera; con una fuerte estabilidad debido a su formación geológica y pendiente menor al $8 \%$; Moderada, sujeta a su pendiente 8 a $25 \%$ y a su formación geológica de moderada estabilidad; y la de Fuerte Susceptibilidad, por desarrollarse en formas fisiográficas, con pendientes superíores al $25 \%$, y suelos de textura arenosa.

Palabras clave: unidades fisiográficas; minerales arcillosos; taxonomía de suelos; susceptibilidad de las tierras; series de suelos.

\begin{abstract}
The objective of this study was to obtain a reliable source of information for the efficient management of existing natural resources. For this, the soils of the area were characterized physically, morphologically and taxonomically. The methodology used was based on the Soil Survey Manual; and the taxonomic classification according to Soil Taxonomy. The multivariate method was also used to determine the susceptibility of soils. Seventeen soil series were identified; moreover, by mineralogical analysis it showed that the presence of the mineral albite (feldspar) predisposes these soils to be less developed in its order, and soils that observed in the presence of rutile (titanium oxide) it indicates very developed soils. Those soils with clay as the montmorillonite and illite are soils with a moderate development. Respect to its land susceptibility, the study area shows three levels: Light, with strong stability due to its geological and slope of less than $8 \%$; Moderate, subject to its slope $8-25 \%$ and its geological formation of moderate stability; and High Susceptibility, developed in landforms with over $25 \%$ slopes and sandy soils.
\end{abstract}

Keywords: physiographic units; clay minerals soil taxonomy; susceptibility of lands; land series.

\section{Introducción}

En la selva peruana, específicamente en la Región Ucayali, existen zonas en la que el verdadero potencial agrícola o el ecoturismo no se conoce. En la actualidad, se ha observado que el conocimiento científico del suelo, indicado en el develamiento de su origen, y desarrollo, es fundamental para el ordenamiento territorial y la planificación económica de cualquier región. Más aún cuando este conocimiento nos revelaría el uso potencial que poseen estos suelos, su patrón de distribución espacial, los factores limitantes para la ejecución de programas y proyectos de desarrollo, así como el empleo de técnicas de

Perú. Email: euclidesraul@gmail.com

2 Profesor principal, Universidad Nacional Agraria La Molina. Lima, Perú. Email: mvalenciar@lamolina.edu.pe

${ }^{3} \mathrm{Ph}$ D. Centro de Investigaciones Fronteras Amazónicas (CIFA-UNU).

manejo adecuadas para su mejor utilización (Cochrane y Sánchez, 1982).

Algunas instituciones como la Universidad Nacional de Ucayali (UNU), el Centro de Investigación de las Fronteras Amazónicas (CIFA), el Gobierno Regional de Ucayali (GOREU) y el Instituto de Investigación de la Amazonia Peruana (IIAP) -Pucallpa, están realizando investigaciones puntuales en la región sobre estos temas, no obstante, las informaciones recopiladas aún son escasas.

Los objetivos fueron: a) Caracterizar física y morfológicamente los suelos, y agruparlos 
taxonómicamente con la finalidad de definir su uso y relacionarlos a su grado de desarrollo; b) Analizar la mineralogía de los suelos y relacionar con su origen y grado de desarrollo; c) Identificar y delimitar la susceptibilidad de los suelos a su deteríoro.

\section{Materiales y métodos}

La zona en estudio se encuentra en la parte media de la cuenca del río Abujao margen derecha del río Ucayali, a 59 kilómetros al sureste de la ciudad de Pucallpa, región Ucayali. Se ubica en los paralelos $8^{\circ} 32^{\prime}$ y $8^{\circ} 45^{\prime}$ LS y los meridianos $73^{\circ} 76^{\prime}$ y $74^{\circ} 04^{\prime}$ 'LW, con un área de 24528,39 hectáreas, dentro de dos zonas de vida: bosque húmedoTropical y bosque muy húmedo - Tropical.

\section{Proceso metodológico}

\section{Recopilación y generación de información temática y cartográfica}

Se recopiló información secundaria de estudios anteríores (ONERN, 1978; Díaz, 2000), información cartográfica, imágenes satelitales Rapideye y del Google Earth Pro, radar SRTM, para proceder a la elaboración del mapa fisiográfico con fines de levantamiento de suelos. Se seleccionaron 40 puntos de muestreo, coordenadas UTM.

\section{Generación de información de campo}

Se realizó el reconocimiento de la zona, para identificar y ajustar los niveles fisiográficos del mapa elaborado en gabinete. En los 40 puntos de muestreo se procedió a la apertura de calicatas hasta la profundidad de 2,00 metros. En ellos se identificaron y caracterizaron los horizontes siguiendo los lineamientos propuestos por el Soil Survey Manual (1993). De cada horizonte se tomaron muestras.

\section{Proceso de análisis de las muestras de suelo}

Las muestras de suelos fueron analizadas en el Laboratorío de Análisis de Suelos, Plantas, Aguas y Fertilizantes (LASPAF) de la Universidad Nacional Agraria La Molina (UNALM). Los parámetros considerados fueron: textura, materia orgánica, $\mathrm{pH}$, fósforo $\mathrm{y}$ potasio disponible, capacidad de intercambio de catiónico (CIC), cationes cambiables.

Una porción de las muestras de suelos de algunos horizontes fue separada y enviada al laboratorío de Difractometría de Rayos X de la Universidad Nacional Mayor de San Marcos para el Análisis Mineralógico mediante Difractómetro de Rayos X en un difractómetro de marca BUKER, modelo D8-FOCUS, el cual empleó un tubo de $\mathrm{Cu}$, cuya longitud de onda correspondiente a $\mathrm{K}-\mathrm{Cu}$ es $=1.5406 \AA$. La selección se realizó con base a su estrecha relación con la litología de la zona.

\section{Clasificación taxonómica de los suelos}

Se siguieron las definiciones y nomenclaturas establecidas en el Keys to Soil Taxonomy (Soil Survey Staff, 2014) hasta el nivel de familia. Se empleó la información mineralógica obtenida, y se relacionó con el desarrollo de los suelos en el área.

\section{Susceptibilidad de los suelos}

Del análisis de las características taxonómicas y ambientales, se derivó el grado de susceptibilidad de los suelos mediante el Sistema de Información geográfica (SIG) y el método del Análisis Multivariable (MINAM, 2011). Se utilizaron los mapas temáticos de suelo y fisiografía obtenidos en el presente estudio, así como los mapas de vegetación, uso actual de la tierra, pendiente y geología de estudios anteríores en la zona. El análisis multivariable consiste en la integración de las características físicas y biológicas con la finalidad de obtener un índice de Susceptibilidad. Se aplicó un modelo matemático denominado "Promedio geométrico ponderado":

$$
\mathrm{S}=\mathrm{Fi}^{*} 0,2+\mathrm{Ge}^{*} 0,2+\mathrm{CoUs} * 0,1+\mathrm{Pe}^{*} 0,2+\mathrm{Pp}^{*} 0,1+\mathrm{Sue}^{*} 0,2
$$

Dónde:

$$
\begin{aligned}
& \mathbf{S}=\text { Susceptibilidad } \mathbf{F i}=\text { Fisiografía } \\
& \text { Ge }=\text { Geología } \quad \text { CoUs }=\text { Cobertura vegetal y Uso de la Tierra } \\
& \mathbf{P e}=\text { Pendiente } \quad \mathbf{p}=\text { Precipitación } \\
& \text { Sue }=\text { Suelo }
\end{aligned}
$$

Para determinar la susceptibilidad de los suelos en el área de estudio, se aplicaron criteríos numéricos o valores según las características que presentan cada variable.

\begin{tabular}{cc}
\hline $1-2$ & Ligera Susceptibilidad \\
\hline $2-3$ & Moderada Susceptibilidad \\
\hline $3-4$ & Fuerte Susceptibilidad \\
\hline
\end{tabular}

\section{Resultados y discusión}

\section{Clasificación Taxonómica}

Se identificaron 17 suelos. Uno pertenece al orden Entisols, trece pertenecen al orden Inceptisols y tres a Ultisols, Tabla 1. Taxonómicamente, el suelo Nejilla pertenece al orden Entisols, por su escaso desarrollo. Por sus características fluvénticas (disminución irregular en el contenido de carbono) ingresa al suborden Fluvents, y al desarrollarse bajo un régimen de humedad údico al gran grupo Udifluvents. Su carencia de características diferenciativas para subgrupo le genera el nombre de Typic Udifluvents.

Los suelos que presentaron un horizonte eluvial, horizonte cámbico, fueron agrupados en el orden Inceptisols. Al desarrollarse bajo un régimen de humedad údico dentro del suborden Udepts y al contener una saturación de bases superíor al $60 \%$ desde los 25 a los 75 centímetros de profundidad al gran grupo de los Eutrudepts, suelos Huasai, Shimbillo, Capirona y Palmera. Si esta condición no se cumple, son calificados dentro de los Dystrudepts: suelos Colina Shebon, Mohena, Santa Rosa, Horizonte, Uvilla, Libertad, Tornillo y Sargento (Soil Survey Staff, 2014). 
Suelos con más desarrollo, presencia de horizonte argílico y una saturación de bases por suma de cationes menor del $35 \%$ pertenecen al orden Ultisols; si se desarrollan bajo un régimen de humedad údico se les califica como sub orden Udults y al no presentar características diferenciativas, para el gran grupo, se les denomina Hapludults. Un horizonte argílico con un espesor menor de 25 centímetros indica el carácter Inceptic del suelo y se les clasifica como subgrupo Inceptic Hapludults, suelo Cedro, mientras los suelos que no presentan otras características diferenciativas como Typic Hapludults, suelos Caoba y Pijuayo (Soil Survey Staff, 2014).

Tabla 1. Unidades Taxonómicas identificadas en el área de estudio

\begin{tabular}{|c|c|c|c|c|}
\hline \multicolumn{4}{|c|}{ Soil Taxonomy (2014) } & \multirow{2}{*}{$\begin{array}{l}\text { Nombre de } \\
\text { los suelos }\end{array}$} \\
\hline Orden & Sub Orden & Gran Grupo & Sub Grupo & \\
\hline Entisols & Fluvents & Udifluvents & $\begin{array}{c}\text { Typic } \\
\text { Udifluvents }\end{array}$ & Nejilla \\
\hline \multirow{13}{*}{ Inceptisols } & \multirow{13}{*}{ Udepts } & \multirow{4}{*}{ Eutrudepts } & \multirow{2}{*}{$\begin{array}{c}\text { Dystric } \\
\text { Fluventic } \\
\text { Eutrudepts }\end{array}$} & Huasai \\
\hline & & & & Shimbillo \\
\hline & & & \multirow{2}{*}{$\begin{array}{c}\text { Dystric } \\
\text { Eutrudepts }\end{array}$} & Capirona \\
\hline & & & & Palmera \\
\hline & & \multirow{9}{*}{ Dystrudepts } & \multirow{6}{*}{$\begin{array}{c}\text { Fluventic } \\
\text { Dystrudepts }\end{array}$} & Colina \\
\hline & & & & Shebon \\
\hline & & & & Mohena \\
\hline & & & & Santa Rosa \\
\hline & & & & Horizonte \\
\hline & & & & Uvilla \\
\hline & & & \multirow{3}{*}{$\begin{array}{c}\text { Typic } \\
\text { Dystrudepts }\end{array}$} & Libertad \\
\hline & & & & Tornillo \\
\hline & & & & Sargento \\
\hline \multirow{3}{*}{ Ultisols } & \multirow{3}{*}{ Udults } & \multirow{3}{*}{ Hapludults } & $\begin{array}{l}\text { Inceptic } \\
\text { Hapludults }\end{array}$ & Cedro \\
\hline & & & \multirow{2}{*}{$\begin{array}{c}\text { Typic } \\
\text { Hapludults }\end{array}$} & Caoba \\
\hline & & & & Pijuayo \\
\hline
\end{tabular}

\section{Densidad Aparente}

La densidad aparente se relaciona con la textura al considerar el volumen total del suelo. La materia orgánica influencia la densidad aparente. Se realizó una de regresión lineal simple para observar la tendencia que existe entre la densidad aparente, el porcentaje de materia orgánica y el porcentaje de arcilla (Buol et al., 1997).

En algunos suelos de la zona de estudio la densidad aparente no se relaciona con la clase textural. En el suelo Nejilla, Figura 1 y Figura 2, se observa que la densidad aparente se incrementa a medida que el contenido de materia orgánica disminuye con la profundidad. Por otro lado, la densidad permanece estable al relacionarlo con la arcilla. Este incremento de la densidad aparente está otorgada por la compactación en el perfil del suelo por las capas menos profundas hacia las más profundas y no por la granulometría del perfil (Vásquez y Tapia, 2005).

En los suelos Shimbillo, Figura 3, Santa Rosa, Figura 4 , se observa similar tendencia con respecto al suelo anteríormente mencionado, en cuanto al incremento de la densidad aparente a medida que decrece el porcentaje de materia orgánica con la profundidad, pero comparando la densidad aparente con el porcentaje de arcilla existe un aumento de la densidad lo cual no sucedió en el suelo anteríor. Es posible. que el movimiento descendente de las partículas de arcillas (proceso de iluviación y eluviación) esté rellenando los espacios porosos de las capas subyacentes, y por consiguiente aumenta su masa manteniendo su mismo volumen (Díaz, 2000).

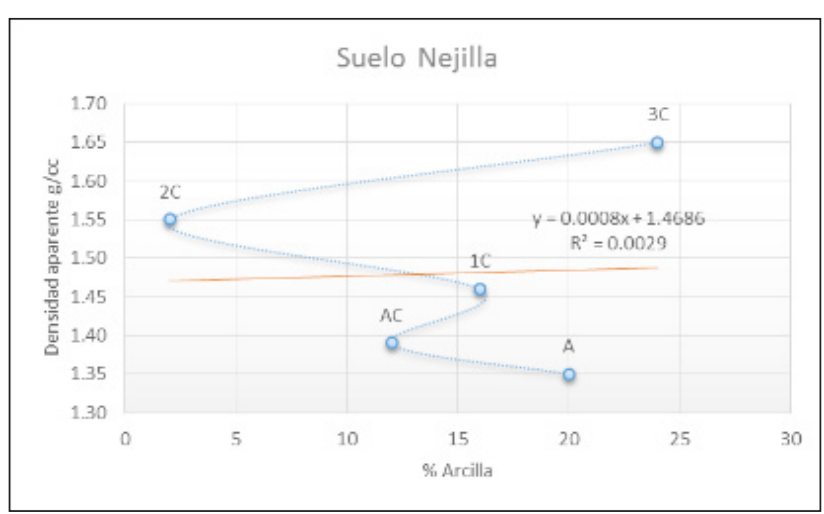

Figura 1. Densidad Aparente vs porcentaje de Materia Orgánica

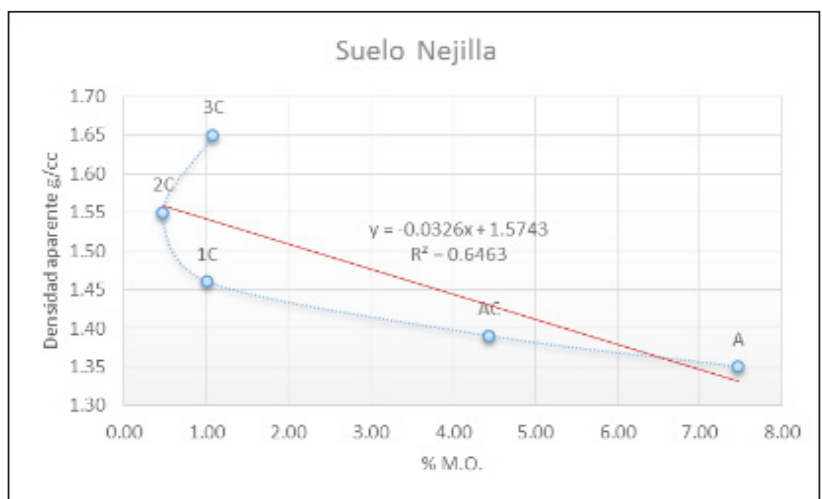

Figura 2. Densidad Aparente vs Porcentaje de Arcilla

En resumen, el aumento de la densidad aparente en las capas más profundas se debería al grado de compactación de los horizontes superíores contra los inferíores y no es un indicador del desarrollo del suelo. Por otro lado, el aumento de la densidad aparente, como producto del movimiento descendente de las partículas de arcillas, sí es un indicador de desarrollo del suelo, esto ocurre especialmente en suelos con presencia de clays skins en las paredes de los peds. En suelos donde exista un buen contenido de arcilla en sus capas profundas y no exista un aumento en su densidad es posible que su desarrollo no se deba al proceso de movimiento descendente de las arcillas por completo si no a otro factor de formación presente en el medio.

Todos los suelos en sus capas superficiales presentan una elevada densidad aparente la cual es debido a la clase textural gruesa a moderadamente gruesa que presentan. 


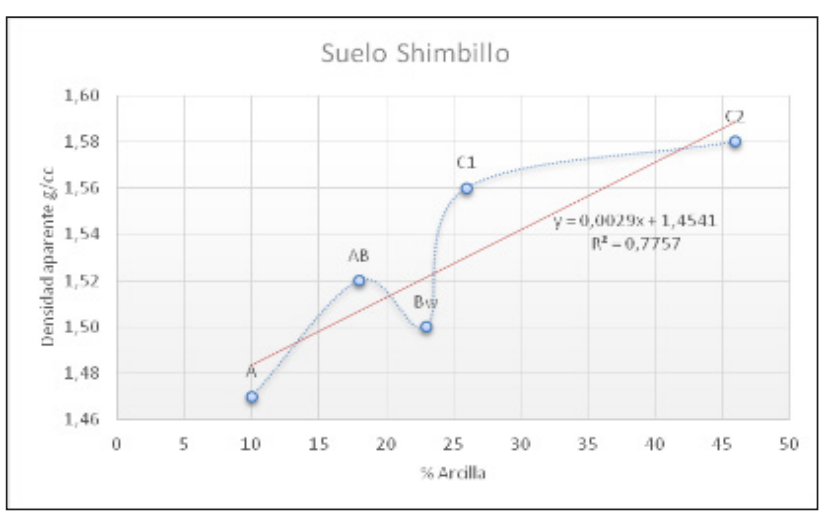

Figura 3. Densidad aparente vs Porcentaje de Arcilla

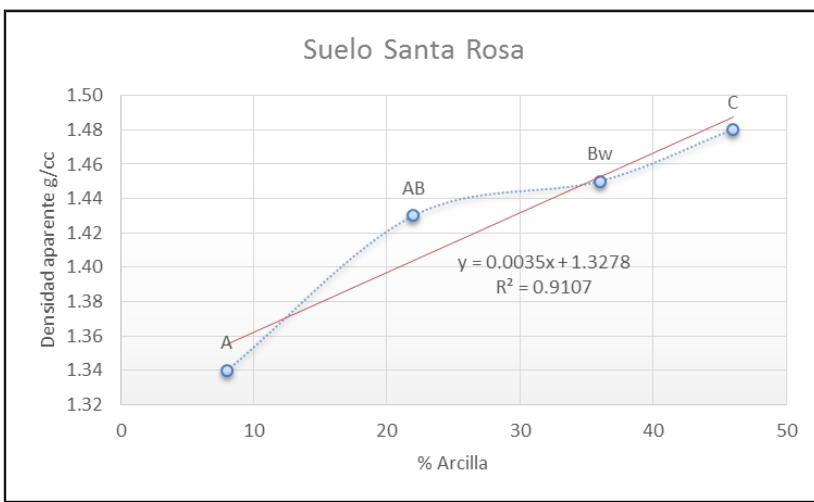

Figura 4. Densidad aparente vs Porcentaje de Arcilla

\section{Valor "n"}

En la Tabla 2 se observa que todos los suelos presentan un valor " $n$ " menor al valor crítico (valor $n \leq 0,7$ ) (Soil Survey Staff, 2014), lo que indica que estos suelos presentan buenas condiciones para el soporte del tránsito y el peso del ganado, además de otras cargas. Asimismo, en todos estos suelos no se presentarán encharcamientos o lámina de agua que signifique la presencia de mal drenaje y la posible degradación o destrucción de su estructura con la pérdida de suelo por erosión laminar.

\section{Familia de Suelos}

Las características que se emplean para identificar familia (Soil Survey Staff, 2014) son de manera secuencial: Clase de Tamaño de Partículas, Clase de Materiales Transportados y/o Alterados por el Hombre, Clase de Mineralogía, Clase de Actividad de Intercambio Catiónico, Clase de Reacción y Calcáreo, Clase de Temperatura del Suelo, Clase por Profundidad del Suelo, Clase por Resistencia a la Ruptura, Clase de Recubrimiento sobre Arenas y Clases de Grietas Permanentes (Soil Survey Staff, 2014).

Los cuatro últimos son dirigidos hacia suelos específicos, los que no se han identificado en el área; además, por tratarse de una zona no intervenida, no existe materiales alterados o transportados por el hombre, mientras que la temperatura, la reacción o $\mathrm{pH}$ y calcáreo del suelo son la mismas para todos los suelos, por lo que la clase de tamaño de partículas, la clase de mineralogía y la clase de actividad de intercambio catiónico son las características para familia de suelos que se analizaron.

Tabla 2. Valor " $n$ " en los 0-30cm del perfil del suelo

\begin{tabular}{|c|c|c|c|c|c|}
\hline Suelos & Hor. & Valor "n" & Suelos & Hor. & Valor "n" \\
\hline \multirow{3}{*}{ Nejilla } & $\mathrm{A}$ & 0,072 & \multirow{2}{*}{ Horizonte } & $\mathrm{A}$ & $-1,221$ \\
\hline & $\mathrm{AC}$ & $-0,076$ & & $\mathrm{Bw}$ & $-0,447$ \\
\hline & $1 \mathrm{C}$ & 0,008 & \multirow{2}{*}{ Mohena } & $\mathrm{A}$ & 0,054 \\
\hline \multirow{2}{*}{ Sargento } & A & $-0,795$ & & $\mathrm{AB}$ & $-0,091$ \\
\hline & $\mathrm{AB}$ & $-1,626$ & \multirow[t]{2}{*}{ Cedro } & $\mathrm{A}$ & $-0,405$ \\
\hline \multirow{3}{*}{ Shebon } & $\mathrm{A}$ & $-0,309$ & & A & $-0,31$ \\
\hline & $\mathrm{Bw}$ & $-0,279$ & \multirow[t]{2}{*}{ Huasai } & BA & $-0,154$ \\
\hline & A & $-0,046$ & & Bw1 & 0,133 \\
\hline \multirow[t]{2}{*}{ Shimbillo } & BA & 0,103 & \multirow{2}{*}{ Caoba } & A & $-0,613$ \\
\hline & $\mathrm{Bw}$ & 0,254 & & $\mathrm{AB}$ & $-0,17$ \\
\hline \multirow{2}{*}{ Uvilla } & A & 0,17 & \multirow{2}{*}{$\begin{array}{l}\text { Tornillo } \\
\text { Libertad }\end{array}$} & $\mathrm{A}$ & $-1,866$ \\
\hline & $\mathrm{Bw}$ & 0,192 & & A & $-0,515$ \\
\hline \multirow{2}{*}{ Santa rosa } & A & $-0,241$ & \multirow{2}{*}{ Capirona } & A & $-0,205$ \\
\hline & $\mathrm{AB}$ & 0,123 & & BA & 0,007 \\
\hline \multirow{4}{*}{ Pijuayo } & A & $-0,092$ & \multirow{2}{*}{ Colina } & $\mathrm{A}$ & $-0,44$ \\
\hline & $\mathrm{AB}$ & 0,094 & & BA & $-0,38$ \\
\hline & & & \multirow{2}{*}{ Palmera } & $\mathrm{A}$ & 0,287 \\
\hline & & & & $\mathrm{Bw}$ & 0,245 \\
\hline
\end{tabular}

Los cuatro últimos son dirigidos hacia suelos específicos, los que no se han identificado en el área; además, por tratarse de una zona no intervenida, no existe materiales alterados o transportados por el hombre, mientras que la temperatura, la reacción o $\mathrm{pH}$ y calcáreo del suelo son la mismas para todos los suelos, por lo que la clase de tamaño de partículas, la clase de mineralogía y la clase de actividad de intercambio catiónico son las características para familia de suelos que se analizaron.

\section{Clase de Tamaño de Partícula}

La clase de tamaño de partículas es aplicada a ciertos horizontes del suelo dentro de límites de profundidad específica, denominada sección de control. Para el caso de los suelos identificados, la sección de control es considerada el espesor entre los $25-100 \mathrm{~cm}$. de profundidad (Soil Survey Staff, 2014).

A continuación se exponen tres suelos modelos que demuestran cómo la distribución del tamaño de partículas puede ser usada para predecir el desarrollo del mismo.

En el suelo Nejilla, un Typic Udifluvents, con una clase textural franca sobre arena franca se observa, Tabla 3 , que existe un buen porcentaje de arena en todos los horizontes del perfil llegando a superar el $70 \%$ en el horizonte $2 \mathrm{C}$, además se observa que no existe un secuencia estable, creciente o decreciente en el porcentaje de arcilla con respecto al incremento de la profundidad lo que demuestra que este suelo no tuvo el suficiente tiempo para desarrollarse y que aún presenta los procesos de acumulación de las 
capas de sedimentos dejados por el río anualmente. La clase de tamaño de partículas es definida para este suelo como franca gruesa.

En este suelo se observa un decrecimiento del contenido de arenas con la profundidad y no existe un proceso claro de eluviación-iluviación de arcillas debido a que el horizonte $\mathrm{C}$ contiene un buen porcentaje de arcillas con respecto al horizonte $2 \mathrm{C}$ y tiene valores erráticos de arcilla en el perfil, además no cumple con los parámetros de acumulación de arcilla para un horizonte argílico y no presenta clays skins (Soil Survey Staff, 2014).

El suelo Cedro, Tabla 5, clasificado a nivel de subgrupo como Inceptic Hapludults, presenta una clase textural en su sección control de franco arcillosa sobre arcilla, su calificación como clase de tamaño de partículas es fina (Soil Survey Staff, 2014).

En este suelo se observa el desarrollo del horizonte argílico, Bt, y la presencia de clays skins en la superficie de los peds, producto de un proceso de iluviación de arcillas. Esto refleja a la distribución del tamaño de partículas que se produce en este pedón como uno de los factores que favorece su desarrollo (Buol, 1997). No obstante, el escaso espesor (menor de 25 centímetros) del horizonte argílico indica que es un suelo intermedio entre un Inceptisols y un Ultisols, o un Inceptisols en proceso de desarrollo.
El suelo Mohena, Tabla 4, un Fluventic Dystrudepts, presenta una clase textural franco arcillo arenosa sobre arcilla, esta última con un espesor de 82 centímetros pedón. Su calificación es de clase de tamaño de partícula fina (Soil Survey Staff, 2014).

\section{Clase de Mineralogía}

Se identificaron los siguientes minerales arcillosos: Montmorillonita (MgO- $\left.\mathrm{Al}_{2} \mathrm{O}_{3.5} \mathrm{SiO}_{2}-\mathrm{xH}_{2} \mathrm{O}\right)$ filosilicato arcilla expandible $(2: 1)$, Caolinita $\left(\mathrm{Al}_{2}\left(\mathrm{Si}_{2} \mathrm{O}_{5}\right)(\mathrm{OH})_{4}\right)$ filosilicato arcilla no expandible (1:1), $\underline{\text { Illíta }}\left(\mathrm{KAl}_{2}\left(\mathrm{Si}_{3} \mathrm{AlO}_{10}\right)\right.$ $(\mathrm{OH})_{10}$ ) filosilicato mica arcilla no expandible $(2: 1)$, y no arcillosos, Cuarzo $\left(\mathrm{SiO}_{2}\right)$ Oxido de Sílice, $\underline{\text { Rutilo }}\left(\mathrm{TiO}_{2}\right)$ Óxido de Titanio, y $\underline{\text { Albita }}\left(\mathrm{Na}\left(\mathrm{AlSi}_{3} \mathrm{O}_{8}\right)\right.$ ) Feldespato sódico (Dana - Hurlbut, 1960).

La mayoría de los suelos presenta un alto contenido de cuarzo $\left(\mathrm{SiO}_{2}\right)$, por el tipo de material geológico presente. Capas de areniscas fomentan la presencia de este mineral cuarzoso, producto de la meteorización acelerada roca en presencia de las condiciones de trópico (Bensoain, 1970; Romero, 1985).

\section{La Mineralogía como Indicador de la Edad de los Suelos}

Los suelos Uvilla Figura 5 y Caoba Figura 6 presentan, según Foth (1990), un desarrollo moderado, debido a sus

Tabla 3. Distribución de tamaño de partículas Suelo Nejilla

\begin{tabular}{|c|c|c|c|c|c|c|c|c|c|c|}
\hline \multirow[b]{2}{*}{ Suelo } & \multirow[b]{2}{*}{ Horz. } & \multirow[b]{2}{*}{$\begin{array}{l}\text { Prof. } \\
(\mathrm{Cm})\end{array}$} & \multicolumn{5}{|c|}{ Arena } & \multirow[b]{2}{*}{$-\underset{(005-0,002)}{\text { Limo }}$} & \multirow[b]{2}{*}{$\begin{array}{c}\text { Arcilla } \\
(<0,002)\end{array}$} & \multirow[b]{2}{*}{$\begin{array}{c}\text { Clase } \\
\text { Textural }\end{array}$} \\
\hline & & & $\begin{array}{c}\text { Muy Gruesa } \\
(2,0-1,0)\end{array}$ & $\begin{array}{c}\text { Gruesa } \\
(1,0-0,5)\end{array}$ & $\begin{array}{c}\text { Media } \\
(0,5-0,25)\end{array}$ & $\begin{array}{c}\text { Fina } \\
(0,25-0,1)\end{array}$ & $\begin{array}{c}\text { Muy Fina } \\
(0,1-0,05)\end{array}$ & & & \\
\hline \multirow{5}{*}{ NEJILLA } & A & 00-07 & 0,04 & 0,12 & 1,22 & 26,10 & 16,52 & 36 & 20 & $\mathrm{Fr}$ \\
\hline & $\mathrm{AC}$ & $07-16$ & 0,00 & 0,04 & 0,76 & 28,46 & 20,74 & 38 & 12 & $\mathrm{Fr}$ \\
\hline & $1 \mathrm{C}$ & $16-32$ & 0,00 & 0,08 & 2,04 & 31,50 & 16,38 & 34 & 16 & $\mathrm{Fr}$ \\
\hline & $2 \mathrm{C}$ & $32-81$ & 0,00 & 0,02 & 1,66 & 51,34 & 24,98 & 20 & 2 & $\mathrm{AFr}$ \\
\hline & $3 \mathrm{C}$ & $81-108$ & 0,02 & 0,26 & 0,90 & 15,14 & 21,68 & 38 & 24 & $\mathrm{Fr}$ \\
\hline
\end{tabular}

Tabla 4. Distribución del tamaño de partículas Suelo Mohena

\begin{tabular}{|c|c|c|c|c|c|c|c|c|c|c|}
\hline \multirow[b]{2}{*}{ Suelo } & \multirow[b]{2}{*}{ Horz. } & \multirow{2}{*}{$\begin{array}{l}\text { Prof. } \\
(\mathrm{Cm})\end{array}$} & \multicolumn{5}{|c|}{ Arena } & \multirow[b]{2}{*}{ Limo } & \multirow[b]{2}{*}{$\underset{(<0,002)}{\text { Arcilla }}$} & \multirow[b]{2}{*}{$\begin{array}{c}\text { Clase } \\
\text { Textural }\end{array}$} \\
\hline & & & $\begin{array}{c}\text { Muy Gruesa } \\
(2,0-1,0)\end{array}$ & $\begin{array}{c}\text { Gruesa } \\
(1,0-0,5)\end{array}$ & $\begin{array}{c}\text { Media } \\
(0,5-0,25)\end{array}$ & $\begin{array}{c}\text { Fina } \\
(0,25-0,1)\end{array}$ & $\begin{array}{c}\text { Muy Fina } \\
(0,1-0,05)\end{array}$ & & & \\
\hline \multirow{6}{*}{ Mohena } & $\mathrm{A}$ & $0-6$ & 0,00 & 1,34 & 4,28 & 29,5 & 8,88 & 36 & 20 & $\mathrm{Fr}$ \\
\hline & $\mathrm{AB}$ & $6-20$ & 0,00 & 0,68 & 6,64 & 42,40 & 16,28 & 24 & 10 & FrA \\
\hline & $\mathrm{Bw}$ & $20-48$ & 0,00 & 0,16 & 4,90 & 36,82 & 10,12 & 26 & 22 & FrArA \\
\hline & $\mathrm{BC}$ & $48-80$ & 0,00 & 0,00 & 1,94 & 13,84 & 10,22 & 26 & 48 & Ar \\
\hline & $1 \mathrm{C}$ & $80-130$ & 0,00 & 0,00 & 0,96 & 14,76 & 4,28 & 24 & 56 & Ar \\
\hline & $2 \mathrm{C}$ & $130-180$ & 0,00 & 1,56 & 4,92 & 20,62 & 12,90 & 28 & 32 & FrAr \\
\hline
\end{tabular}

Tabla 5. Distribución del tamaño de partículas Suelo Cedro

\begin{tabular}{|c|c|c|c|c|c|c|c|c|c|c|}
\hline \multirow[b]{2}{*}{ Suelo } & \multirow[b]{2}{*}{ Horz. } & \multirow{2}{*}{$\begin{array}{l}\text { Prof. } \\
(\mathrm{Cm})\end{array}$} & \multicolumn{5}{|c|}{ Arena } & \multirow{2}{*}{$\begin{array}{c}\text { Limo } \\
(005-0,002)\end{array}$} & \multirow{2}{*}{$\begin{array}{c}\text { Arcilla } \\
(<0,002)\end{array}$} & \multirow{2}{*}{$\begin{array}{c}\text { Clase } \\
\text { Textural }\end{array}$} \\
\hline & & & $\begin{array}{c}\text { Muy Gruesa } \\
(2,0-1,0)\end{array}$ & $\begin{array}{c}\text { Gruesa } \\
(1,0-0,5)\end{array}$ & $\begin{array}{c}\text { Media } \\
(0,5-0,25)\end{array}$ & $\begin{array}{c}\text { Fina } \\
(0,25-0,1)\end{array}$ & $\begin{array}{c}\text { Muy Fina } \\
(0,1-0,05)\end{array}$ & & & \\
\hline \multirow{5}{*}{ Cedro } & A & $0-42$ & 0,38 & 5,48 & 10,82 & 28,94 & 18,38 & 28 & 8 & $\operatorname{FrA}$ \\
\hline & BA & $42-95$ & 0,00 & 0,47 & 5,23 & 13,92 & 21,38 & 27 & 32 & FrAr \\
\hline & $\mathrm{Bt}$ & $95-116$ & 0,00 & 0,00 & 0,84 & 4,88 & 16,28 & 30 & 48 & $\mathrm{Ar}$ \\
\hline & $\mathrm{C} 1$ & $116-139$ & 0,00 & 0,00 & 2,64 & 9,56 & 25,80 & 28 & 34 & FrAr \\
\hline & $\mathrm{C} 2$ & $139-167$ & 0,00 & 1,02 & 4,90 & 17,56 & 16,52 & 26 & 34 & FrAr \\
\hline
\end{tabular}


contenidos de cuarzo y esmectita, así mismo, la albita ha desaparecido del perfil del suelo, y no se observan rastros del óxido de titanio. El suelo Uvilla es el suelo más joven de los Inceptisols y el suelo Caoba el más joven de los Ultisols.

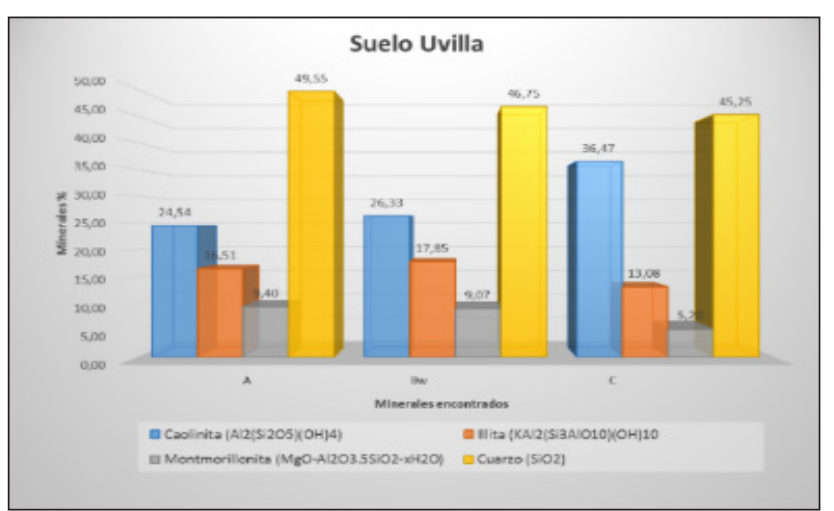

Figura 5. Minerales arcillosos y no arcillosos Suelo Uvilla

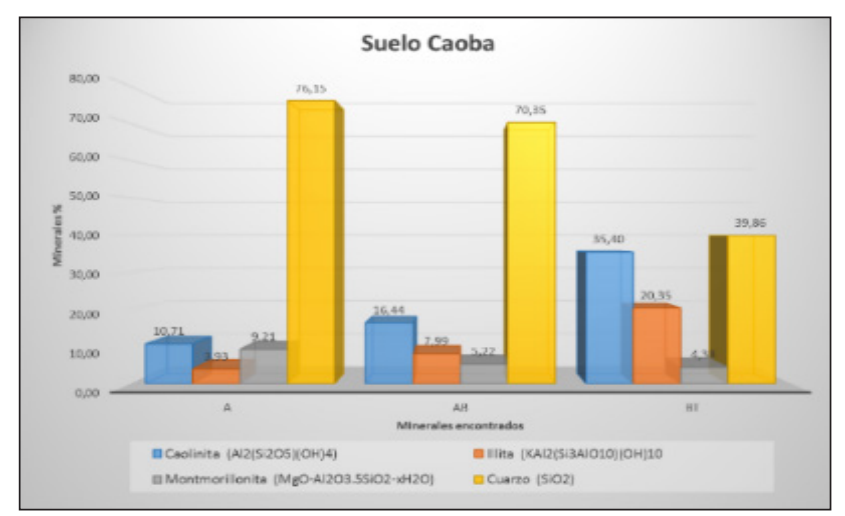

Figura 6. Minerales arcillosos y no arcillosos Suelo Caoba

Los suelos Huasai (Figura 7) y Pijuayo (Figura 8), según Foth (1990), presentan una meteorización intensa debido a que la albita ha desaparecido del perfil y se encuentran altos porcentajes de rutilo que es un indicador de un suelo evolucionado (envejecido). Debe resaltarse, la acumulación de caolinita, que es un indicador que el mineral albita se ha meteorizado (Foth, 1990). Dentro del Orden Inceptisols, el suelo Huasai es el más desarrollado, al igual que el suelo Pijuayo lo es para el Orden Ultisols.

El suelo Palmera, Figura 9, es un suelo que se desarrolló in situ y exhibe una moderada meteorización, muestra en su composición mineralógica un $4 \%$ de albita llegando incluso a superar el $19 \%$ en los horizontes más profundos; estos suelos presentan una erosión fuerte debido a que el porcentaje de la caolinita no se acumula en los horizontes superficiales como en los otros suelos siendo esto producto de la meteorización de la albita.

Aparentemente, en esta zona existe un sistema doble, es decir, los suelos envejecen y rejuvenecen al mismo tiempo.
$\mathrm{Su}$ parte superficial expuesta a los procesos o factores ambientales se va meteorizando y se va acumulando el rutilo, un indicador de suelos muy evolucionados (Foth, 1990).

Al estar estos suelos en pendientes superíores al $25 \%$ existe la probabilidad de erosión en la parte superficial, produciéndose pérdidas cada vez mayores por efecto de las lluvias intensas y la gravedad. Esta pérdida ocasiona que se exponga material parental menos evolucionado presencia de mineral albita, y así el ciclo nuevamente se inicia.

Se puede predecir que el suelo Nejilla, un Entisols (Figura 10), evolucionó sobre el material procedente del suelo Palmera que se depositó en esta zona fisiográfica de terrazas bajas y medias, producto de la erosión, transporte y sedimentación en épocas anteríores, puesto que se evidencia la existencia de minerales como la albita y el rutilo.

Para determinar las Clases de Mineralogía, se utiliza la misma sección control utilizada para las Clases de Tamaño de Partículas. Taxonómicamente, todos los suelos a excepción del suelo Santa Rosa (Esmectitica) presentan una mineralogía mezclada. En la Figura 11, se presenta un mapa con la distribución de los minerales arcillosos y no arcillosos identificados en la zona.

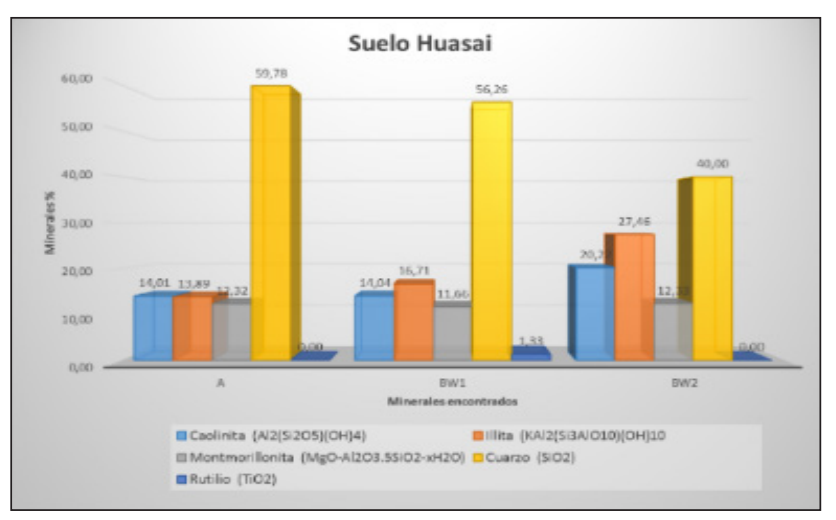

Figura 7. Minerales arcillosos y no arcillosos Suelo Huasai

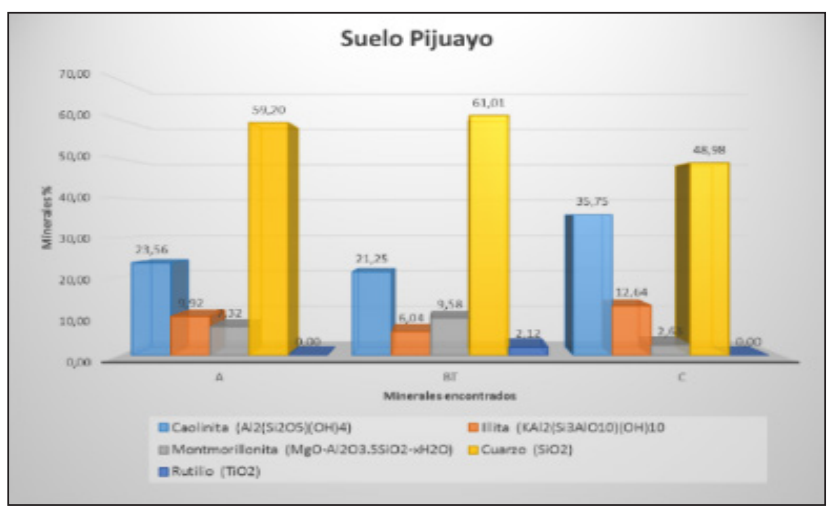

Figura 8. Minerales arcillosos y no arcillosos Suelo Pijuayo 


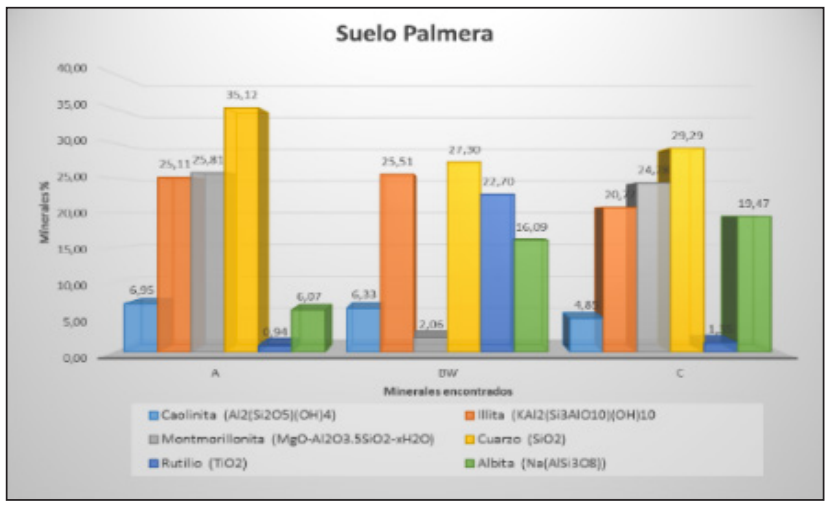

Figura 9. Minerales arcillosos y no arcillosos Suelo Palmera

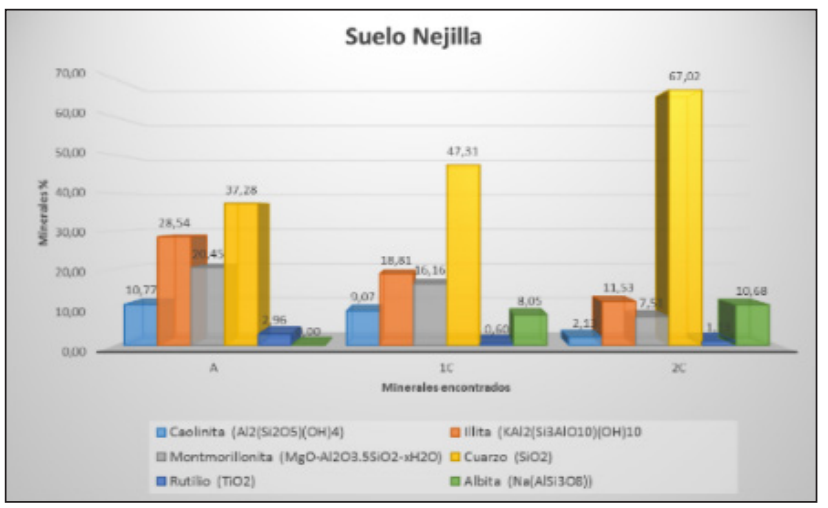

Figura 10. Minerales arcillosos y no arcillosos Suelo Nejilla

\section{Clase de Actividad de Intercambio Catiónico}

En el suelo Nejilla (Figura 12) la CIC se incrementa al aumentar los contenidos de arcilla y materia orgánica, por lo que su CIC está influenciada por las cargas permanentes (sustitución isomórfica) y por las dependientes del $\mathrm{pH}$, otorgando a estos suelos los enlaces de adsorción para los elementos nutricionales. Su clase de actividad de intercambio de cationes es clasificada como Superactiva.

En el suelo Santa Rosa (Figura 13) la CIC aumenta conforme el contenido de arcilla se incrementa, por lo que, la CIC está influenciada por las cargas producidas por sustitución isomórfica. Su clase de actividad de intercambio catiónico es activa.

En el suelo Pijuayo (Figura 14) existe una disminución de la CIC con respecto al porcentaje de minerales arcillosos, por lo que la CIC depende no solo de las cargas permanentes, sino también de las cargas dependientes del $\mathrm{pH}$ generadas por la materia orgánica. Su clase actividad de intercambio catiónico es calificado como semiactiva.

\section{Susceptibilidad de las Tierras}

Según el MINAM ((2011) en la cuenca media del río Abujao, se encuentran tres niveles de susceptibilidad (Figura 15), Las tierras con susceptibilidad ligera, representan el 5,17 $\%$ (1267,71 ha). La fuerte estabilidad que presenta se basa en que está conformada por suelos de terrazas medias, con pendientes que van desde 0 a $8 \%$ y localizadas sobre bosques primaríos, secundaríos o en algunas purmas. Todo tipo de bosques produce una buena resistencia a la degradación por erosión debido a la cubierta de raíces que sujetan al suelo, incrementándose esta resistencia cuando la pendiente tiende a $0 \%$. Se correlaciona con la unidad geológica de Depósitos aluviales subrecientes, al no recibir materiales nuevos por los ríos.

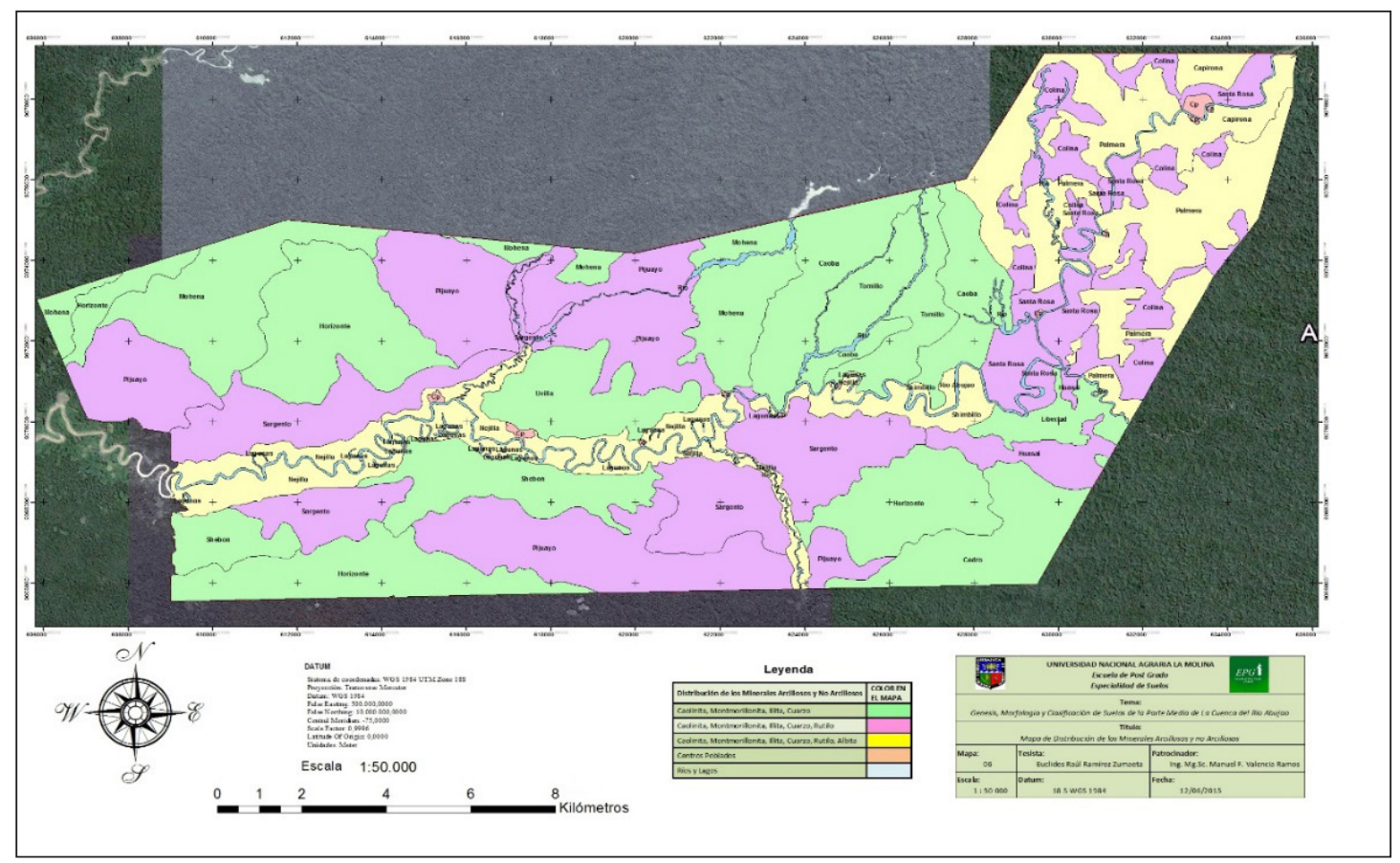

Figura 11. Mapa de Distribución de Minerales Arcillosos y No Arcillosos en los Suelos Identificados en la Zona 


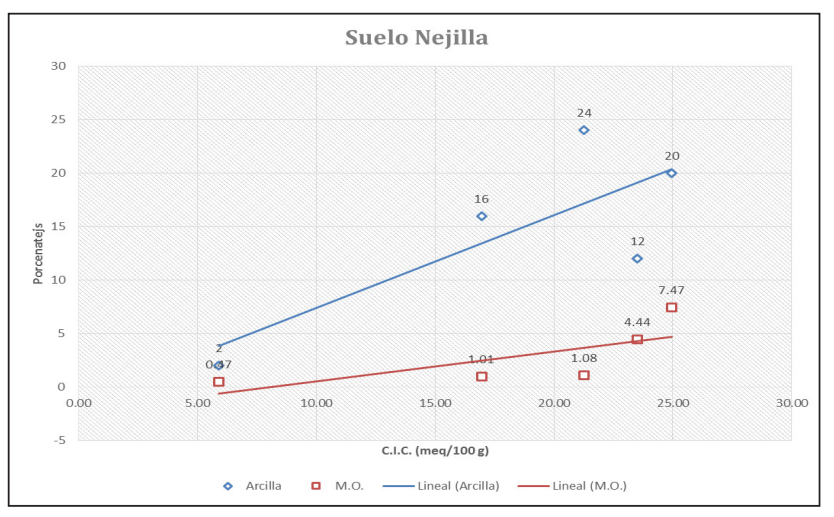

Figura 12. Clase de AIC del Suelo Nejilla

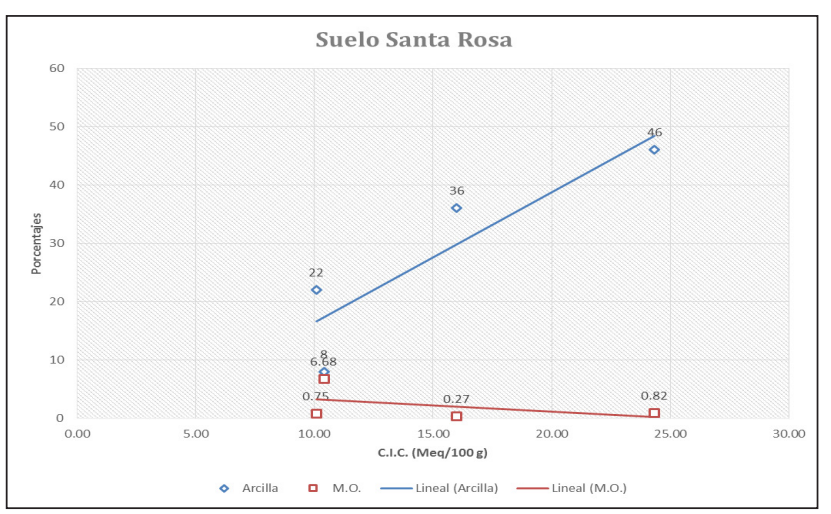

Figura 13. Clase de AIC del Suelo Santa Rosa.

Las tierras con susceptibilidad moderada, representan el 79,12 \% (19 406,57 ha). Su moderada estabilidad se basa en la clase textural gruesa a moderadamente gruesa que presentan los suelos identificados, así como a las elevadas precipitaciones de la zona y la pendiente en que se encuentran, 8-25\%, lo que genera procesos de erosión ligera a moderada y pérdida del material edáfico. Se correlaciona con unidades geológicas, de baja estabilidad (Depósitos aluviales recientes) y de moderada estabilidad (Formación Ipururo). La susceptibilidad es generada por el tipo de material litológico que presentan (De la Cruz et al., 1997).

Las tierras con susceptibilidad fuerte, comprende el 13,22 \% (3 242,99 ha). La baja estabilidad es producto de la fisiografía colinosa y de sus pronunciadas pendientes que llegan a superar el $25 \%$, acentuada con clases texturales de gruesa a moderadamente gruesa, produciéndose en gran medida eventos erosivos que son maximizados por las elevadas precipitaciones del lugar; asimismo, la baja estabilidad geológica de la formación Chambira determina que estas zonas tengan una fuerte susceptibilidad.

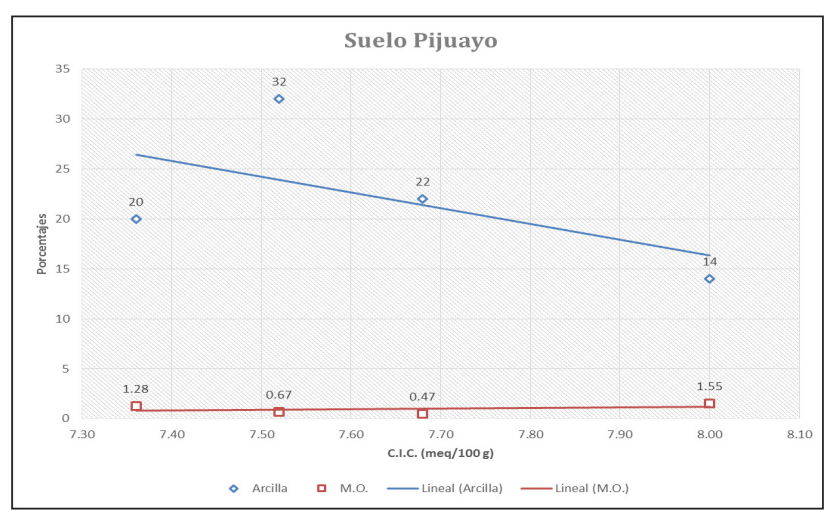

Figura 14. Clase de AIC del Suelo Pijuayo.

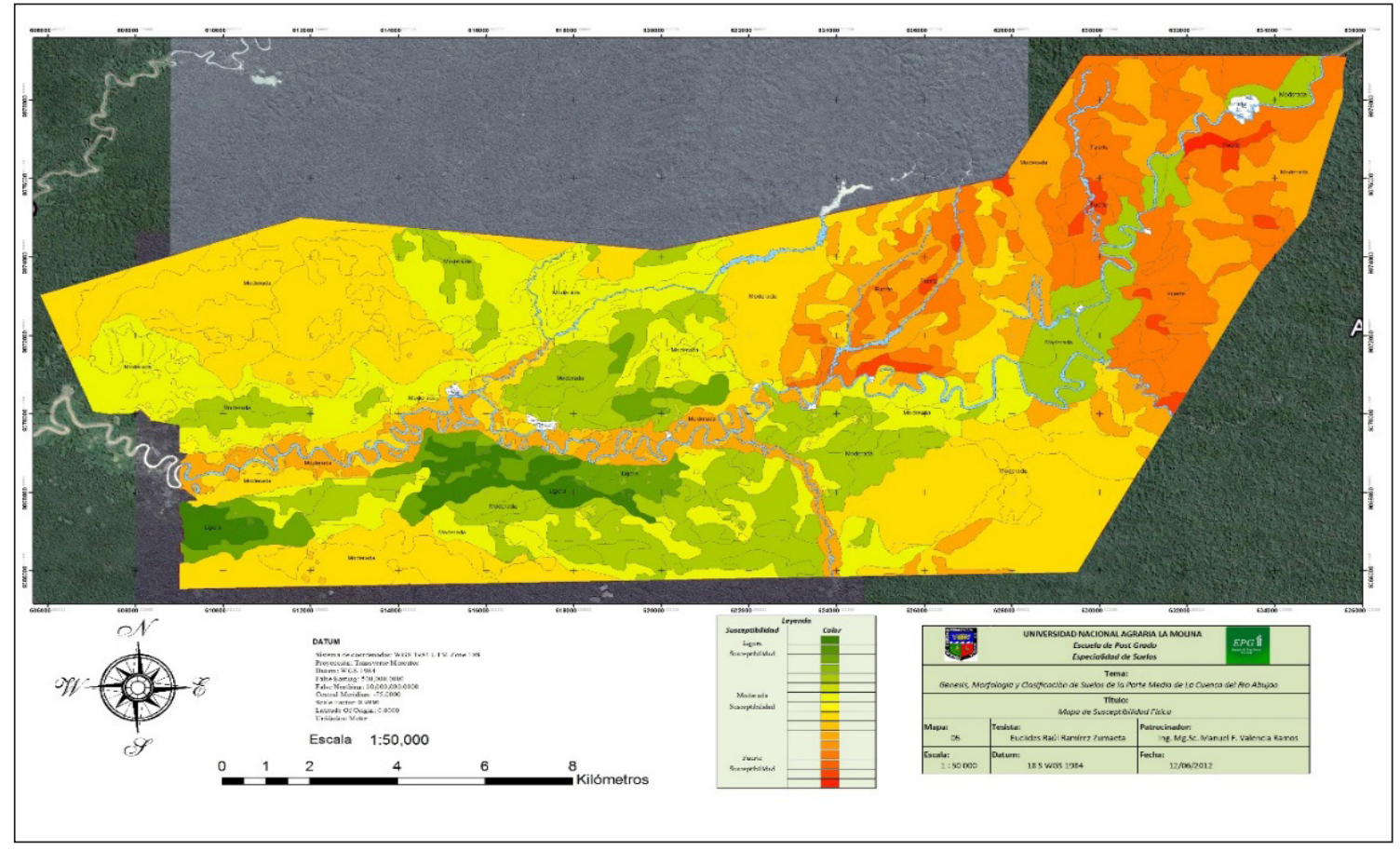

Figura 15. Mapa de Susceptibilidad Física 


\section{Conclusiones}

Se identificaron 17 suelos, la mayoría pertenece al Orden Inceptisols, suelos de mediano desarrollo y un horizonte cámbico, De ellos, solo cuatro presentan una fertilidad potencial adecuada, indicado por un alto porcentaje de saturación de bases y nula posibilidad de encharcamiento al poseer un valor " $n$ " menor al valor crítico 0,7 . La densidad aparente no guarda relación con la textura, sus valores son más altos, al disminuir el volumen, por la compactación de los horizontes superíores sobre los inferíores. El origen y grado de desarrollo de estos suelos se ha establecido en base a la estabilidad de los indicadores; caolinita o arcilla tipo 1:1, la albita, un feldespato de sodio, y el rutilo, un óxido de titanio. Los suelos más jóvenes dentro de los Inceptisols, suelo Uvilla, presentan altos valores se albita y bajos de caolinita. En los Ultisols, suelos Caoba y Pijuayo, la albita ha desaparecido y su desarrollo está asociado a la arcilla caolinita. El contenido de rutilo en el suelo Huasai (Inceptisols) y en el suelo Pijuayo (Ultisols) indican su mayor antigüedad. Del estudio, se deriva que en la zona se encuentran, principalmente, suelos de moderada susceptibilidad a la erosión, por su textura moderadamente gruesa, presencia de altos valores de albita, bajos de rutilo y su localización en pendientes de 8-25\%.

\section{Literatura citada}

Bensoain, E. 1970. Mineralogía de Suelos Curso. Instituto Interamericano de Ciencia Agrícola de la OEA. Centro de Enseñanza e Investigación Tropical. Torrialba Costa Rica. 233-293p.

Buol, S.W.; Hole, F.D. y Mc Cracken. 1997. Génesis y Clasificación de suelos. Primera Edición en español. Ed. Trillas S.A. México.

Cochrane, T.T. y Sanchez, P.A. 1982. Recursos de Tierras, Suelo y su Manejo en la Región Amazónica. En Amazonia: Investigación sobre Agricultura y Uso de Tierras. CIAT 035, 4 (82): $141-218$.

Dana - Hurlbut. 1960. Manual de Mineralogía. Segunda Edición. Buenos Aires.

De la Cruz, B.; Natalio; Zedano, J. et al. 1997. "Geología de los Cuadrángulos de San Roque, Río Callería, San Lucas, Pucallpa, Nuevo Utiquinia, Cantangallo, y Divisor Yurúa Ucayali”. Boletín No 102, Serie A: Carta Geológica Nacional. INGEMMET.

Díaz, Z. E. 2000. Génesis, Morfología y Clasificación de Algunos Suelos de Pucallpa. Tesis para optar el grado de Magister Sciencie en Suelos. Universidad Nacional Agraria La Molina. Lima, Perú.

Foth, D.H. 1990. Fundamentals of Soil Science, Octava edición. Michigan State University. 382 p.

INRENA. 1995. Mapa Ecológico del Perú. Guía Explicativa. Segunda Ed. Instituto Nacional de Recursos Naturales. Lima, Perú.

MINAM, 2011. Mapa de Vulnerabilidad Física del Perú "Herramienta para la gestión de Riesgo". Primera edición. Lima - Perú

ONERN. 1978. Inventarío, Evaluación e Integración de los Recursos Naturales de la Zona de Pucallpa - Abujao. Oficina Nacional de Recursos Naturales. Lima, Perú.

Romero, F.R. et. al. 1985. Determinación de familias mineralógicas en suelos sobre rocas graníticas de la provincia de la Coruña. Cuaderno Lab. Xeoloxico de Luxe. Coruñas. 10: 171-190.

Soil Survey Staff. 2014. Keys to Soil Taxonomy. Natural Resources Conservation Service. USA. Departament of Agriculture. Twelfth Edition. 305 p.

Soil Survey Division Staff. 1993. Soil Survey Manual. Soil Conservation Service. U.S. Department of Agriculture Handbook, 18. 315p.

Vásquez, F. y Tapia, R. 2005. Edafología y Climatología Forestal; Universidad de Huelva; España. 